\title{
Beneficial Rhizobacterium Bacillus amyloliquefaciens SQR9 Induces Plant Salt Tolerance through Spermidine Production
}

\author{
Lin Chen, ${ }^{1}$ Yunpeng Liu, ${ }^{2}$ Gengwei Wu, ${ }^{1}$ Nan Zhang, ${ }^{1}$ Qirong Shen, ${ }^{1}$ and Ruifu Zhang ${ }^{1,2}$ \\ ${ }^{1}$ Jiangsu Provincial Key Lab for Organic Solid Waste Utilization, National Engineering Research Center for Organic-based \\ Fertilizers, Jiangsu Collaborative Innovation Center for Solid Organic Waste Resource Utilization, Nanjing Agricultural \\ University, Nanjing, 210095, P.R. China; and ${ }^{2}$ Key Laboratory of Microbial Resources Collection and Preservation, Ministry \\ of Agriculture, Institute of Agricultural Resources and Regional Planning, Chinese Academy of Agricultural Sciences, \\ Beijing 100081, P.R. China
}

Accepted 11 March 2017.

\begin{abstract}
The inoculation of plants with plant-growth-promoting rhizobacterium has been an effective strategy for enhancing plant salt tolerance to diminish the loss of agricultural productivity caused by salt stress; however, the signal transmitted from bacteria to the plant under salt stress is poorly understood. In this study, the salt tolerance of Arabidopsis thaliana and Zea mays was enhanced by inoculation with Bacillus amyloliquefaciens SQR9. Using dialysis bags with different molecular weight cutoffs, we sorted through the molecules secreted by SQR9 and found that spermidine is responsible for enhancing plant salt tolerance. An SQR9 $\triangle$ speB mutant deficient in spermidine production failed to induce plant salt tolerance. However, the induction of plant salt tolerance was disrupted by mutating genes involved in reduced glutathione (GSH) biosynthesis and the salt overly sensitive pathway in Arabidopsis. Using quantitative real-time polymerase chain reaction, this study demonstrated that spermidine produced by SQR9 leads to increased glutamine synthetase and glutathione reductase gene expression, leading to increased levels of GSH, which is critical for scavenging reactive oxygen species. SQR9-derived spermidine also upregulates the expression of $\mathrm{NHX1}$ and $\mathrm{NHX} 7$, which sequesters $\mathrm{Na}^{+}$into vacuoles and expels $\mathrm{Na}^{+}$from the cell, thereby reducing ion toxicity.
\end{abstract}

Salt stress is one of the most critical agricultural problems (Shabala and Cuin 2008). Excess salinity imposes nutrient deficiency, osmotic stress, oxidative stress, and ion toxicity on plants and, consequently, leads to inhibition of plant growth and even death (Chinnusamy et al. 2006). Therefore, it is necessary to improve plant salt tolerance to enhance agricultural production.

Plant tolerance to salt stress is mainly mediated through hormone regulation, osmotic homeostasis, detoxification, and ionic homeostasis (Deinlein et al. 2014). High salinity disrupts plant phytohormone homeostasis, and hormone regulation is necessary

L. Chen and Y. Liu contributed equally to this article.

Corresponding author: R. Zhang; Telephone: 86-25-84396477;

Fax: 86-25-84396260; E-mail: rfzhang@njau.edu.cn

*The $\boldsymbol{e}$-Xtra logo stands for "electronic extra" and indicates that one supplementary table and eleven supplementary figures are published online.

(c) 2017 The American Phytopathological Society for controlling plant growth, stomatal closure, and the expression of several phytohormone-dependent genes (Dinneny et al. 2008; Geng et al. 2013). The accumulation of osmoprotectants reduces water loss, maximizes water uptake, and mitigates osmotic stress induced by salinity (Székely et al. 2008; Upadhyay et al. 2012). Antioxidants can scavenge reactive oxygen species (ROS), which would be overproduced during salt stress and cause plant programmed cell death, thereby protecting the plant from the damage caused by salt stress (Meyer et al. 2007; Mittler and Blumwald 2015; Moschou et al. 2008;). Moreover, some active transporters in the membrane, such as $\mathrm{Na}^{+} / \mathrm{H}^{+}$antiporter (NHX) and high-affinity $\mathrm{K}^{+}$transporter $(\mathrm{HKT})$, can aid in salt tolerance by transporting $\mathrm{Na}^{+}$out of the cytoplasm (Deinlein et al. 2014; Zhu 2001).

Several bacteria that colonize plant roots were found to benefit plants through complex interactions and, therefore, were termed plant-growth-promoting rhizobacteria (PGPR). Plant roots can release specific compounds such as malic acid to attract PGPR (Fan et al. 2012; Rudrappa et al. 2008). Under stress, PGPR can induce chemical and physical changes in the plant to help plants against abiotic stress, called induced systemic tolerance (Dimkpa et al. 2009; Ma et al. 2014; Yang et al. 2009).

Volatile organic compounds (VOC) emitted from Bacillus subtilis GB03 have been reported to trigger induced systemic tolerance in plants (Zhang et al. 2008, 2010). VOC emitted from GB03 were able to facilitate shoot to root $\mathrm{Na}^{+}$recirculation and reduce $\mathrm{Na}^{+}$import in Arabidopsis roots by regulating the transcription of the HKT1 in shoots and roots of Arabidopsis. Under osmotic stress, phosphoethanolamine N-methyltransferase (PEAMT) expression was upregulated by VOC emitted from GB03, which increased glycine betaine and choline synthesis in Arabidopsis, helping the plants tolerate osmotic stress via an abscisic acid (ABA)-independent pathway (Liu and Zhang 2015; Zhang et al. 2010). Other molecules produced by PGPR strains that confer plant tolerance to abiotic stress include 1-aminocyclopropane-1carboxylate (ACC) deaminase, which degrades the ethylene precursor ACC (Dimkpa et al. 2009; Glick et al. 2007; Mayak et al. 2004); trehalose, which regulates carbon and nitrogen metabolism (Suárez et al. 2008); and indole-3-acetic acid (IAA) and cytokinin, which regulate genes involved in stress tolerance by maintaining plant hormone homeostasis (Dodd and Pérez-Alfocea 2012; Yang et al. 2009). In short, organic compounds produced by PGPR regulate plant photosynthesis, hormone homeostasis, osmotic homeostasis, cell membrane integrity, detoxification, and ionic homeostasis to confer plant salt tolerance. 
The mechanisms allowing plants to tolerate salt stress are very complicated. New mechanisms and genes involved in plant salt tolerance are constantly reported. Thus, other unknown mechanisms involved in PGPR-induced plant salt tolerance may exist. Our previous studies revealed that $B$. amyloliquefaciens SQR9, a well-studied PGPR strain, enhanced plant salt tolerance by regulating phytohormone, osmolyte, antioxidant, and ion content in plants (Chen et al. 2016). In contrast with the known mechanisms by which PGPR induce plant salt tolerance, this study showed that spermidine produced by SQR9 confers plant salt tolerance in the two following ways: by regulating glutamine synthetase (GS) and glutathione reductase (GR) expression to increase reduced glutathione (GSH) levels, thus alleviating ROS damage; and by regulating $\mathrm{Na}^{+} / \mathrm{H}^{+}$antiporters in the tonoplast $(\mathrm{NHX} 1)$ and $\mathrm{Na}^{+} / \mathrm{H}^{+}$ antiporters in the plasma membrane (NHX7) expression and activating salt overly sensitive (SOS) proteins to control $\mathrm{Na}^{+}$ content in plants.

\section{RESULTS}

The molecular weight of the SQR9-secreted signal that confers plant salt tolerance is between 100 and $500 \mathrm{Da}$.

Under salt stress, SQR9 inoculation increased the number of Arabidopsis lateral roots but not primary root length (Supplementary Fig. S1) and enhanced plant growth (Fig. 1). SQR9 inoculation increased photosynthetic rate and reduced ROS (e.g., $\mathrm{H}_{2} \mathrm{O}_{2}$ ) content (Supplementary Fig. S2). Previous studies showed that SQR9 conferred plant salt tolerance by inhibiting the increase of ABA, reducing $\mathrm{Na}^{+}$content, and enhancing total soluble sugar (TSS) and GSH content as well as catalase (CAT) and peroxidase activity in the plant (Chen et al. 2016). However, the signal released by SQR9 that affects plant salt tolerance pathways was not clearly determined. To identify the molecular signal produced by SQR9 and the pathways affected by this signal, SQR9 was wrapped in dialysis bags of different molecular weights and tested for the ability to induce plant salt tolerance. This allowed us to determine the range of the molecular weight of the signal. The fresh weight and height of plants inoculated with SQR9 were higher than plants inoculated with inactive SQR9 (negative control). The biomass of plants treated with SQR9 wrapped in dialysis bags with a molecular weight cut-off (MWCO) greater than or equal to $500 \mathrm{Da}$ was higher than the negative control (Fig. 1A, B, and $\mathrm{C}$ ). There was no significant difference between plants treated with SQR9 wrapped in 100-Da MWCO dialysis bags and the negative control. The results obtained with Arabidopsis were similar to those obtained with maize. We checked the growth of SQR9 in 100- and 500-Da dialysis bags in the rhizosphere and they showed similar growth curves (Supplementary Fig. S3). Leaf chlorophyll was tested as an indicator of plant phenotype, and low leaf chlorophyll content was used as indicator of impaired plants. The trend of leaf chlorophyll content was similar to plant biomass (Fig. 1D). These results indicate that the molecular weight of the SQR9-derived signal that effectively confers plant salt tolerance is between 100 and $500 \mathrm{Da}$.
A

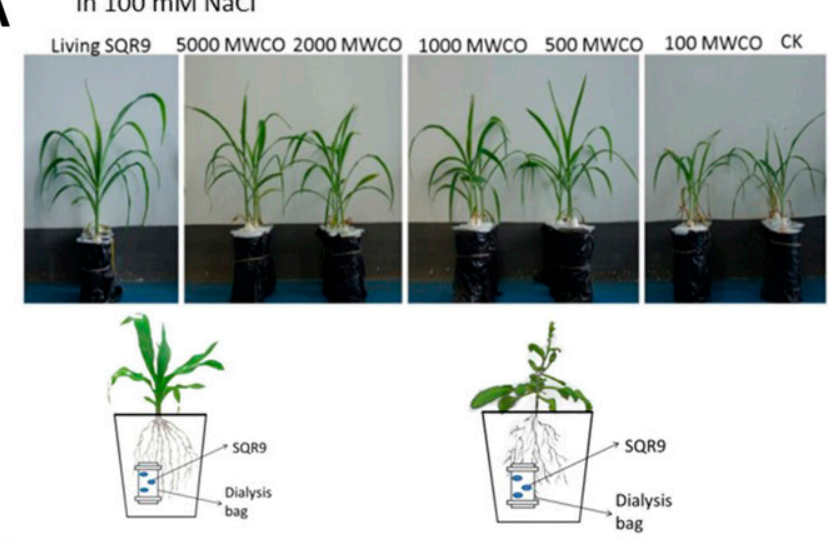

C

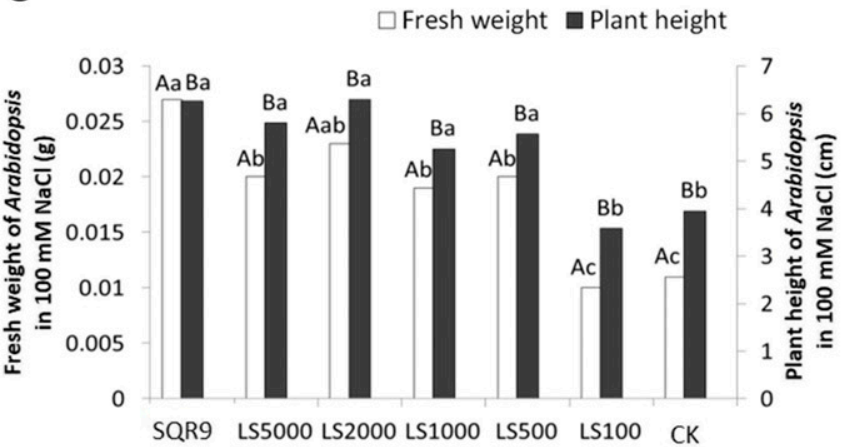

Treatments
B

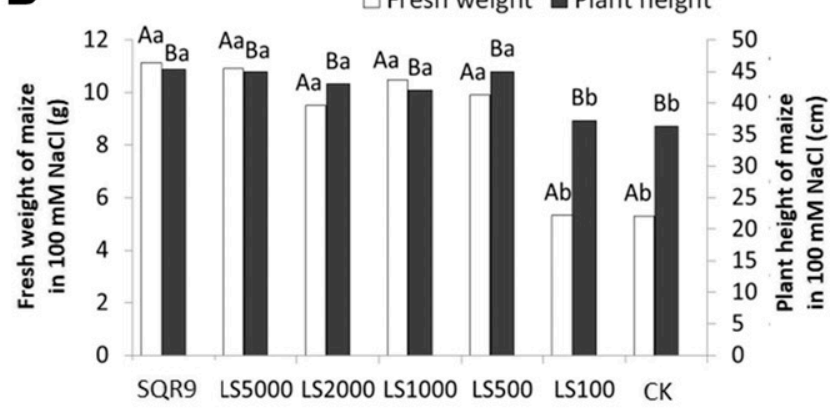

Treatments

D

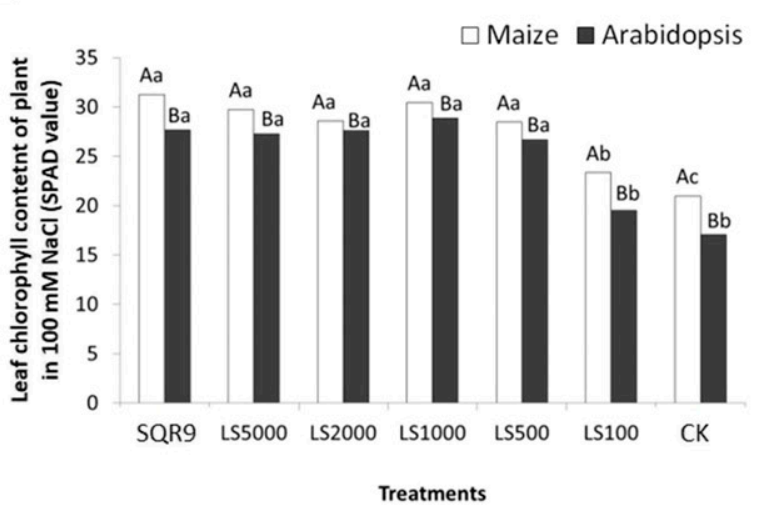

Fig. 1. Effects of Bacillus amyloliquefaciens SQR9 secretions of different molecular weights on plant salt tolerance. A, Representative images of plants treated with SQR9 wrapped in different molecular weight cut-off dialysis bags $(5,000,2,000,1,000,500$ and $100 \mathrm{Da})$. B, Quantification of maize fresh weight and plant height. C, Quantification of Arabidopsis fresh weight and plant height. White and black bars represent plant fresh weight and plant height, respectively. D, Chlorophyll content (SPAD value) was measured. Salt concentration was $100 \mathrm{mM} \mathrm{NaCl}$. SQR9 treatment = plants inoculated by SQR9 without dialysis bag (positive control); CK = negative control; and LS5000, LS2000, LS1000, LS500, and LS100 = living SQR9 wrapped in 5,000-, 2,000-, 1,000-, 500-, and 100-Da dialysis bags, respectively. White and black bars represent maize and Arabidopsis, respectively. Mean values in the same column with different letters indicate significant differences (Duncan's least significant difference, $P \leq 0.05, n=21$ for maize, and $n=48$ for Arabidopsis). 
The signal molecule confers plant salt tolerance by regulating GSH and $\mathrm{Na}^{+}$contents.

Our previous study showed that many physiological indexes of plants inoculated with SQR9 are affected, including ABA, TSS, CAT, and peroxidase activity, as well as GSH and $\mathrm{Na}^{+}$ content (Chen et al. 2016). To confirm which primary mechanisms for plant salt tolerance were induced by the secreted signal (100 to $500 \mathrm{Da}$ ), the physiological indicators of various pathways that could be affected by SQR9 inoculation were measured in the presence or absence of SQR9-secreted molecules between 100 to 500 Da. GSH content in maize and Arabidopsis inoculated with SQR9 or SQR9 wrapped in 500-Da dialysis bags was significantly higher than plants treated with inactive SQR9 or SQR9 wrapped in a 100-Da MWCO dialysis bag (Fig. 2A and B). However, ABA content and TSS content in both Arabidopsis and maize were not affected by SQR9-secreted molecules between 100 and 500 Da (Supplementary Fig. S4A, B, C, and D). In Arabidopsis, CAT activity was higher in plants inoculated with SQR9 wrapped in 500-Da dialysis bags than in that with 100-Da dialysis bags; however, the same experiments in maize did not show significant difference between the 100- and 500-Da treatment. Furthermore, the $\mathrm{Na}^{+}$content in maize and Arabidopsis inoculated with SQR9 or SQR9 wrapped in 500-Da MWCO dialysis bags was significantly decreased compared with plants inoculated with inactive SQR9 or SQR9 wrapped in a 100-Da MWCO dialysis bag (Fig. 2C and D). However, the $\mathrm{Na}^{+}$content in a maize shoot (stem and leaf) inoculated with SQR9 or SQR9 wrapped in 100- and 500-Da MWCO dialysis bags was significantly decreased compared with plants inoculated with inactive SQR9 (Fig. 2C). This result indicated that one or more compounds with a molecular weight smaller than $100 \mathrm{Da}$ were involved in reducing $\mathrm{Na}^{+}$content in shoots. These results revealed that an SQR9-secreted molecule with a molecular weight between 100 and 500 Da confers plant salt tolerance by enhancing the GSH content in plants, reducing the $\mathrm{Na}^{+}$content in Arabidopsis and roots of maize, and enhancing CAT activity in Arabidopsis.

\section{Verification of the major plant salt tolerance pathway induced by SQR9.}

To confirm the roles of CAT, GSH, and ion homeostasis in SQR9-induced plant salt tolerance, Arabidopsis mutants deficient in CAT, the GSH metabolic pathway, and the SOS pathway were inoculated with SQR9. The cat2-1 mutation plant deficient in CAT activity showed salt tolerance responding to SQR9 inoculation as well as the wild-type plant (Supplementary Fig. S5). The $g s$ and $g r$ mutations deficient in glutamate-cysteine ligase and GR, respectively, were sensitive to salt stress in the presence of SQR9, indicating that reduced GSH is involved in SQR9-dependent salt stress tolerance (Fig. 3; Supplementary Fig. S6). Mutation in $h k t l$, which is involved in $\mathrm{Na}^{+}$circulation from shoot to root and $\mathrm{Na}^{+}$transportation into the plant cell, did not alter the response of plants to SQR9 under salt stress (Fig. 3). NHX1 and NHX7 (SOS1) encode $\mathrm{Na}^{+}$transporters located at the vacuolar membrane and plasma membrane, respectively, and control the $\mathrm{Na}^{+}$dynamic in plant cells. Deletion of either of these
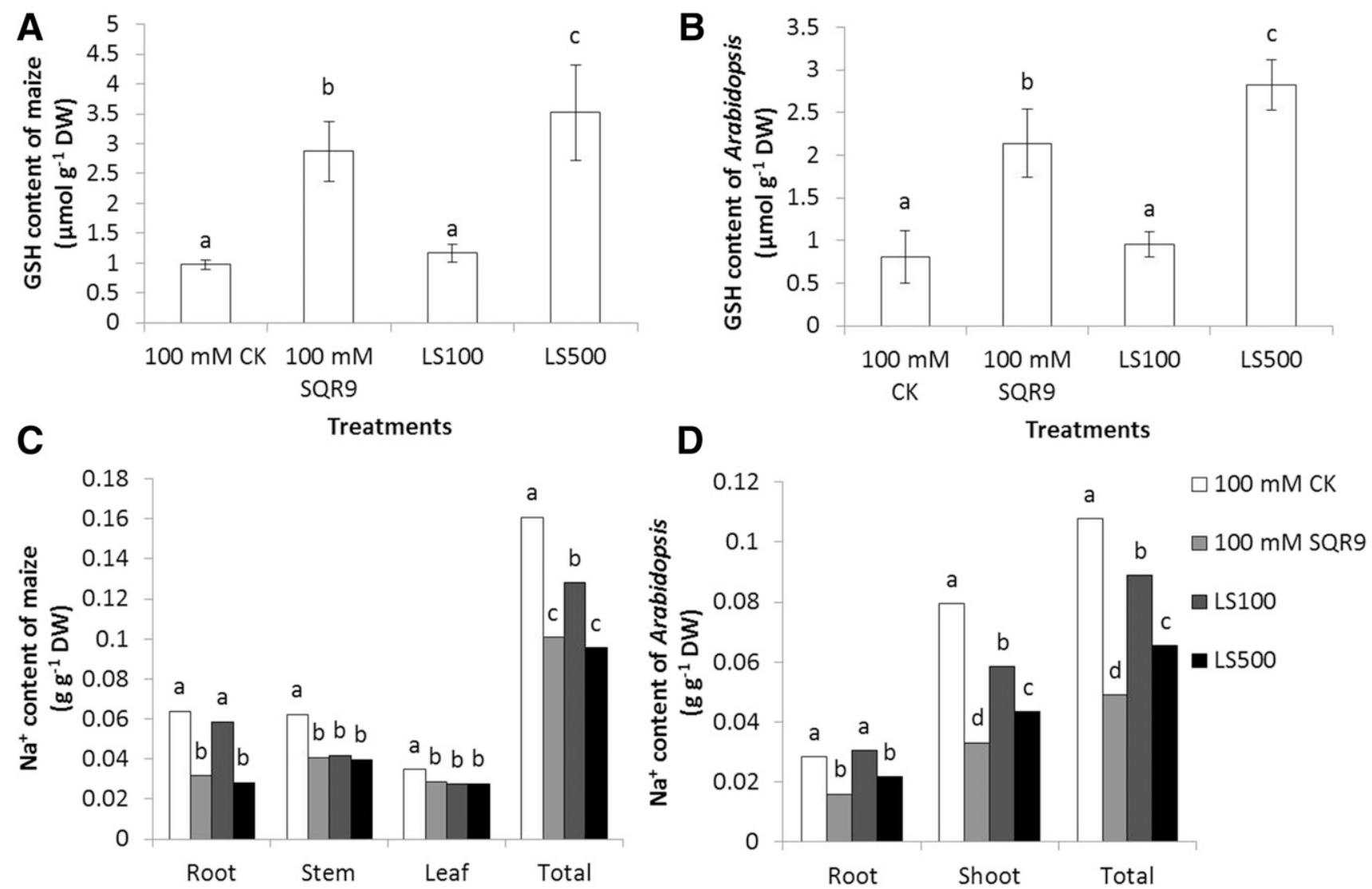

Fig. 2. Effects of Bacillus amyloliquefaciens SQR9 secretions of different molecular weights on reduced glutathione (GSH) content and $\mathrm{Na}^{+}$content of plants. A, GSH content and $\mathbf{C}, \mathrm{Na}^{+}$content in maize were measured after maize was treated with B. amyloliquefaciens SQR9 secretions of different molecular weights under salt stress for 20 days. B, GSH content and $\mathbf{D}, \mathrm{Na}^{+}$content were measured in Arabidopsis after being similarly treated for 14 days. Columns: $100 \mathrm{mM} \mathrm{CK}=$ plants treated with inactivated SQR9 under $100 \mathrm{mM} \mathrm{NaCl}$ (negative control) and $100 \mathrm{mM}$ SQR9 = plants inoculated with SQR9 under $100 \mathrm{mM} \mathrm{NaCl}$ (positive control). Different letters indicate significant differences between treatments (Duncan's least significant difference test, $P \leq 0.05, n=5$, mean \pm standard deviation). 
two genes resulted in the loss of SQR9-induced plant tolerance to salt stress. In addition, mutation of $\operatorname{sos} 2$ or $\operatorname{sos} 3$, which aid in regulating the $\mathrm{Na}^{+}$content in the cytoplasm, resulted in the loss of SQR9-induced plant tolerance to salt stress (Fig. 3). The GSH metabolic pathway was related to ROS but not $\mathrm{Na}^{+}$content in plants; thus, the $\mathrm{Na}^{+}$content of $g s$ and $g r$ mutants was still reduced significantly with inoculation of SQR9. Meanwhile, the $\mathrm{Na}^{+}$content of $h k t 1$ mutants was reduced by SQR9 significantly, which was similar to that of wild-type plants. However, there was no significant difference between the $\mathrm{Na}^{+}$ content of $\operatorname{sos} 1, \operatorname{sos} 2, \operatorname{sos} 3$, and $n h x 1$ mutants inoculated with SQR9 and the control under salinity (Supplementary Fig. S7). These results showed that GSH synthesis (controlled by GS and GR) and the SOS pathway (controlled by NHX1, SOS1, SOS2, and SOS3) are necessary for SQR9 to trigger plant salt tolerance.

\section{The SQR9-produced signal that confers} plant salt tolerance is spermidine.

To identify the signal responsible for conferring plants tolerance to salt stress, the 100- to 500-Da SQR9 secretions were extracted with different organic solvents. Under salt stress, the plant fresh weight increased when treated with SQR9 secretions from the aqueous, ethyl ether, and especially chloroform extracts (Fig. 4). However, the root fresh weight had no significant difference and even significantly decreased when treated with the SQR9 secretion extracted with ethyl acetate and n-pentane, respectively (Fig. 4). These results reveal that the key signal molecule displayed the highest solubility in chloroform phase but was insoluble in ethyl acetate and n-pentane. The SQR9-derived molecules between the molecular weights of 100 and 500 Da were classified. Base on the knowledge of chemicals with activity to affect plant physiology, IAA, gibberellin acid 3 (GA3), transzeatin (ZT), trehalose, and some types of polyamines (PA) were selected as potential signals with a molecular weight between 100 and 500 . These potential signals were tested for solubility in the aforementioned extraction reagents. IAA, GA3, ZT, and trehalose are poorly soluble in chloroform. However, PA displayed the greatest solubility in chloroform compared with the other reagents; as a consequence, they are considered to be potential signals secreted by SQR9 for conferring plant salt tolerance. PA produced by
SQR9 were detected by high-performance liquid chromatography (HPLC). Spermidine was detected in both extracellular and intracellular samples of SQR9, and the content was 26.4 and $29.9 \mu \mathrm{M}$, respectively, in the bacterial culture (optical density at $600 \mathrm{~nm}\left[\mathrm{OD}_{600}\right]=1$ ), whereas spermine was not detected (Fig. $5 \mathrm{~A}, \mathrm{~B}$, and $\mathrm{C}$ ). The B. amyloliquefaciens SQR9 genome was searched for genes related to PA synthesis, and $s p e B$ was found to be the key gene in PA biosynthesis (Fig. 5D). The $s p e B$ gene was knocked out, and the mutant strain was complemented with the entire $s p e B$ gene. The mutation of $s p e B$ caused a deficit in extraand intracellular spermidine production. The extracellular and intracellular spermidine contents for mutant-complemented strain $\Delta$ speB (c- $\Delta$ speB) were 22.2 and $26.6 \mu \mathrm{M}$, respectively, in the bacterial culture $\left(\mathrm{OD}_{600}=1\right)($ Fig. $5 \mathrm{C})$. There was no significant difference between the growth rate of $\Delta s p e B, \mathrm{c}-\Delta s p e B$, and wild-type SQR9 strains in either Luria-Bertani (LB) medium or Murashige and Skoog (MS) medium (with or without $100 \mathrm{mM} \mathrm{NaCl}$ )

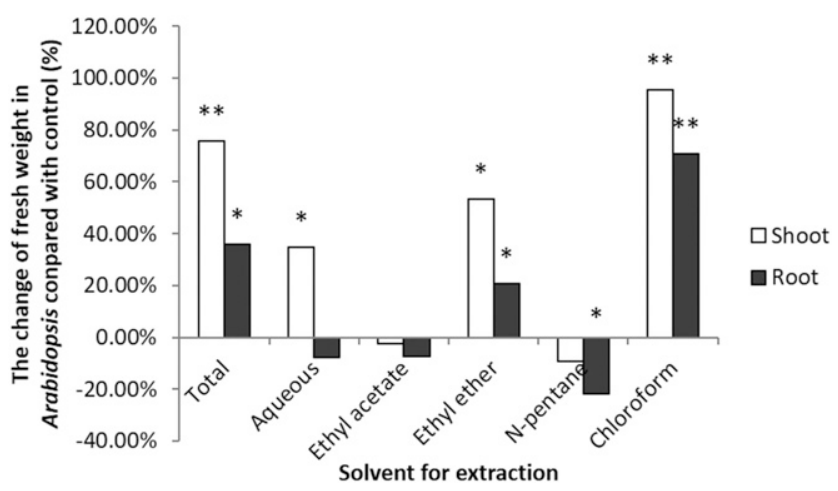

Fig. 4. Effects of various SQR9 secretion extracts on plant biomass under salt stress. SQR9 secretions and Landy medium (control) were extracted with aqueous, ethyl acetate, ethyl ether, n-pentane, and chloroform phases. Values indicate the changes induced by SQR9 secretions extracted with different phases compared with the Landy medium control. Asterisks indicate statistically significant differences between the control and plants treated with SQR9 secretions ( $t$ test, $n=3$, mean \pm standard deviation); * and $* *$ indicate $P \leq 0.05$ and 0.01 , respectively.
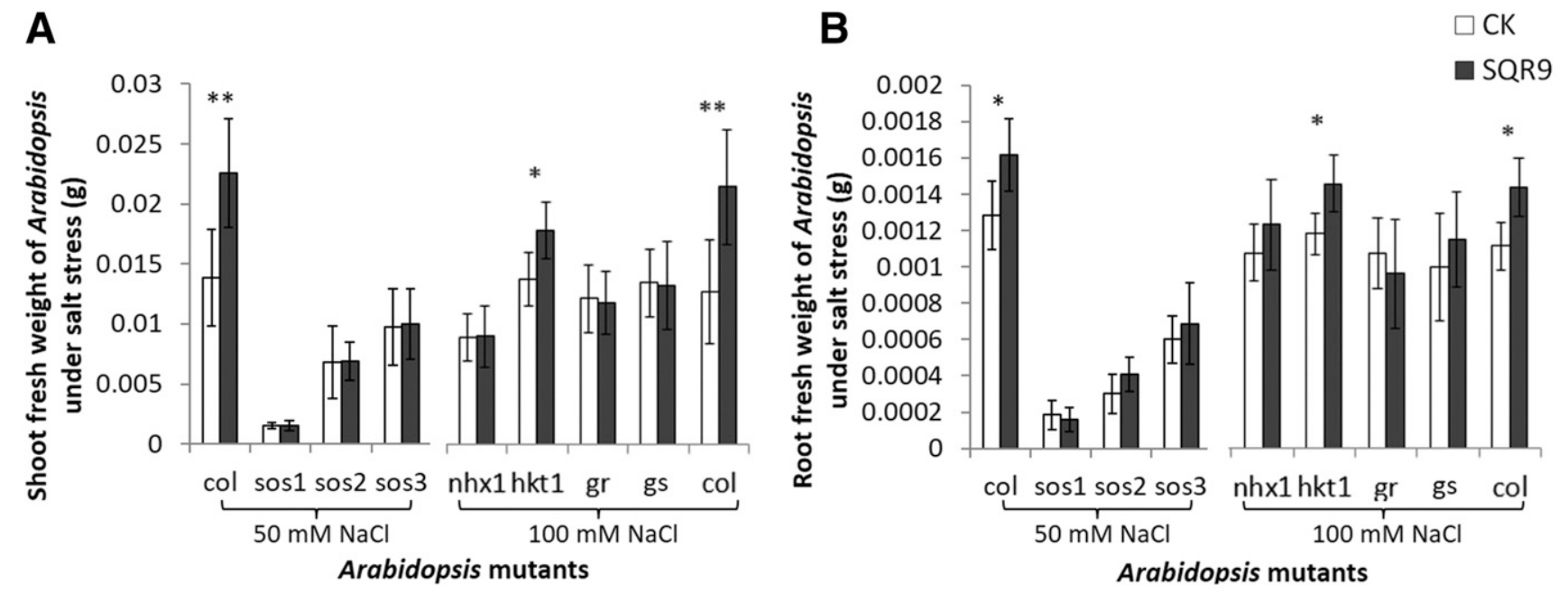

Fig. 3. Effects of Bacillus amyloliquefaciens SQR9 on the response of Arabidopsis mutants to salt stress. The first five mutants (sos1, sos2, sos3, nhx1, and $h k t 1)$ are missing genes related to ion balance in the plant. The last two mutants ( $g r$ and $g s$ ) are involved in reduced glutathione synthesis. Different Arabidopsis mutants were inoculated with SQR9 or heat-inactivated SQR9 as a control. A, Plant shoot fresh weight and B, root fresh weight were measured under salt stress for 14 days. Black and white bars represent SQR9 and heat-inactivated SQR9 control treatments, respectively. Values are the mean \pm standard deviation from 48 replicates. Columns: col = wild-type Arabidopsis; sos 1 , sos 2, and sos 3 mutants were treated with $50 \mathrm{mM} \mathrm{NaCl}$; and $n h x 1, h k t 1, g r$, and $g s$ mutants were treated with $100 \mathrm{mM} \mathrm{NaCl}$. Asterisks indicate statistically significant differences between plants inoculated with SQR9 and control plants $(t$ test); * and ** indicate $P \leq 0.05$ and 0.01 , respectively. 
(Supplementary Fig. S8). These results revealed that speB gene knock out did not affect SQR9 growth.

\section{SQR9-produced spermidine induced plant salt tolerance.}

To study the role of spermidine in inducing plant salt tolerance, plants growing on plates were treated with different concentrations of spermidine under salt stress. The optimal concentration of spermidine that effectively enhances plant salt tolerance is between 1 and $10 \mu \mathrm{M}$ (Fig. 6A). The speB gene was the key gene of synthesis of putrescine and spermidine (Fig. 5D). To determine whether spermidine was the key SQR9 signal, dicyclohexylamine (DCHA), an inhibitor of spermidine synthase, was used to inhibit the production of spermidine by SQR9 (Fig. 6B) (Biondi et al. 1988). The addition of DCHA resulted in an inability of SQR9 to confer plant salt tolerance (Fig. 6C). Furthermore, the mutant strain $\Delta s p e B$ did not aid plants against salt stress but promoted Arabidopsis growth in the absence of salt stress (Fig. 6D; Supplementary Fig. S9). As expected, complementation strain c- $\Delta$ speB conferred the same level of plant salt tolerance as the wild-type SQR9 (Fig. 6E). Moreover, the deficiency of the $s p e B$ mutant strain in conferring plant salt tolerance was rescued by purified spermidine $(5 \mu \mathrm{M})$ (Supplementary Fig. $\mathrm{S} 10)$. These results indicate that SQR9-produced spermidine plays a vital role in inducing plant salt tolerance.

\section{Spermidine confers plant salt tolerance through regulating $\mathrm{GSH}$ and $\mathrm{Na}^{+}$contents.}

We found that the 100- to 500-Da compounds secreted by SQR9 affected plant GSH and SOS pathway to induce plant salt tolerance in this study; therefore, we wanted to check whether spermidine functioned through regulating GSH and $\mathrm{Na}^{+}$content. Transcription levels of GS, GR, NHX1, and NHX7 were examined in Arabidopsis upon inoculation with or addition of SQR9, a spermidine-production-deficient mutant, the complemental strains, or pure spermidine. The $G S$ and $G R$ genes encode key enzymes involved in GSH biosynthesis and reduction pathways. $N H X 1$ and $N H X 7$ are the key genes involved in reducing
$\mathrm{Na}^{+}$content in the cytoplasm. Transcription of $G S, G R, N H X 1$ and NHX7 in Arabidopsis was upregulated upon treatment with either pure spermidine or upon inoculation with wild-type SQR9 compared with plants inoculated with inactive SQR9 (Fig. 7). Inoculation with the mutant strain $\triangle s p e B$ did not lead to a significant increase in $G S, G R, N H X 1$, and $N H X 7$ transcription. These results indicate that SQR9-produced spermidine mainly regulated the expression of $G S$ and $G R$ genes which, in turn, enhanced the GSH content in plants. These data further suggest that enhanced transcription of $N H X 1$ and $N H X 7$ by SQR9-derived spermidine might be involved in the mechanism by which SQR9 protects plants against salt stress.

To ensure the independence of spermidine functions on other pathways such as HKT1, the responses of the mutant plants to spermidine were investigated. Results showed that spermidine induced $h k t l$ mutant plant tolerance to excess salt; however, spermidine could no longer induce the tolerance of plants deficient in SOS1 SOS2, SOS3, NHX1, GR, or GS (Fig. 8). These results are consistent with the influence of SQR9 on Arabidopsis mutants and confirm that spermidine induces plant salt tolerance through the SOS pathway and GSH metabolism (Figs. 3 and 8).

\section{DISCUSSION}

Our previous study has demonstrated that SQR9 maintains photosynthesis, reduces $\mathrm{Na}^{+}$content, enhances antioxidant and TSS content, as well as inhibits ABA accumulation in plants to help plants tolerate salt stress (Chen et al. 2016). In this research, we identified the major signal produced and secreted by SQR9, which conferred plant salt tolerance, as spermidine. Moreover, we found that spermidine conferring plant tolerance to salt stress is dependent on regulating the SOS pathway and GSH synthesis, whereas CAT, TSS, and ABA in Arabidopsis and maize are not necessary for the induced tolerance to salt stress by SQR9-produced spermidine. However, the SOS pathway and GSH synthesis are both necessary for SQR9-induced plant salt tolerance. In the present study, SQR9-produced spermidine was
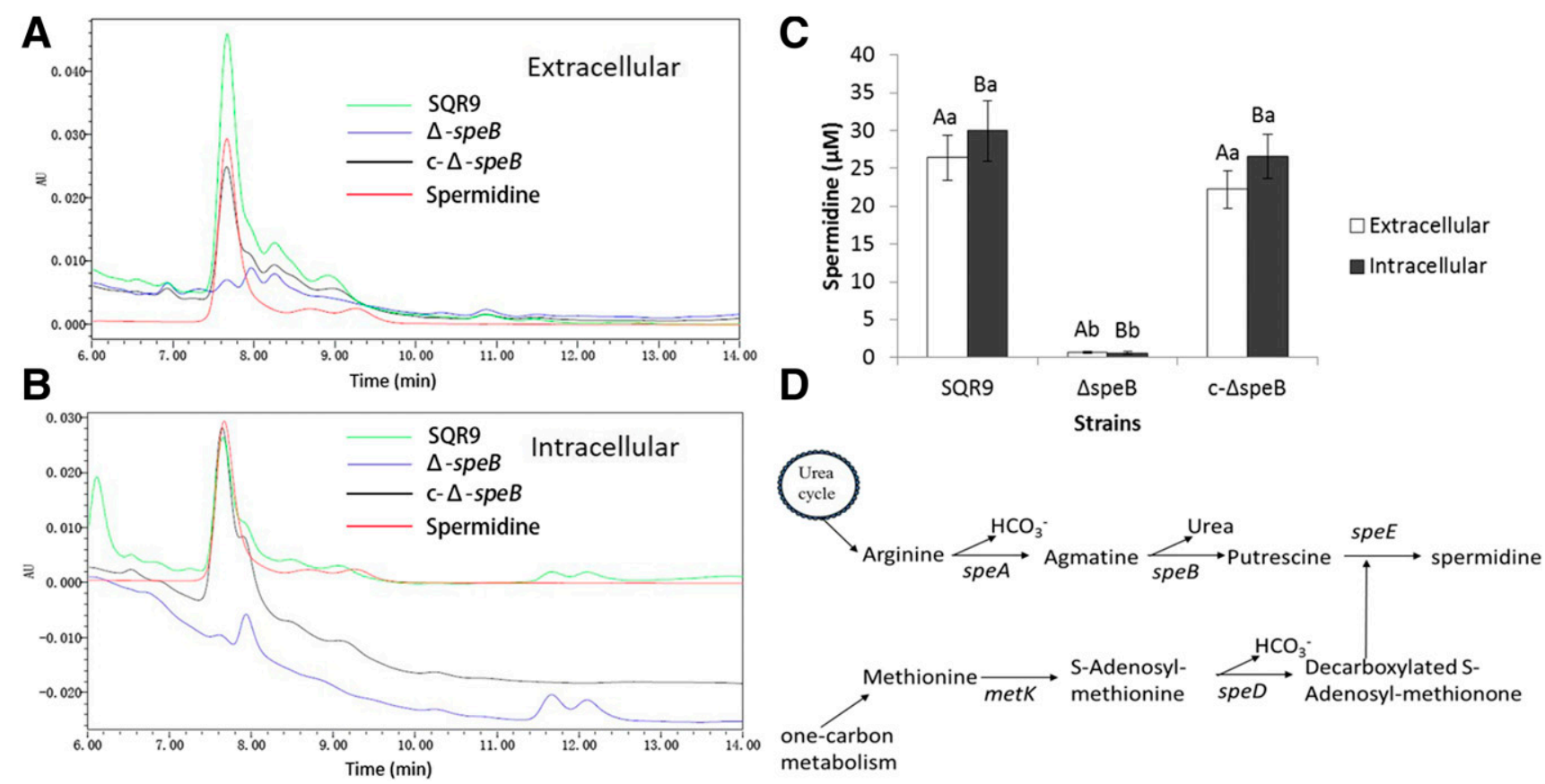

Fig. 5. High-performance liquid chromatography (HPLC) analysis of A, extracellular and $\mathbf{B}$, intracellular spermidine from Bacillus amyloliquefaciens SQR9, $\Delta s p e B$, and c- $\Delta$ speB strains. C, Extracellular and intracellular spermidine content from SQR9, $\Delta s p e B$, and c- $\Delta$ speB strains was quantified. D, Polyamine biosynthetic pathway in $B$. amyloliquefaciens $\mathrm{SQR}$ 9. Values indicate the mean \pm standard error from three replicates. Different letters above the columns indicate statistically significant differences (Duncan's least significant difference test, $P \leq 0.05$ ). 


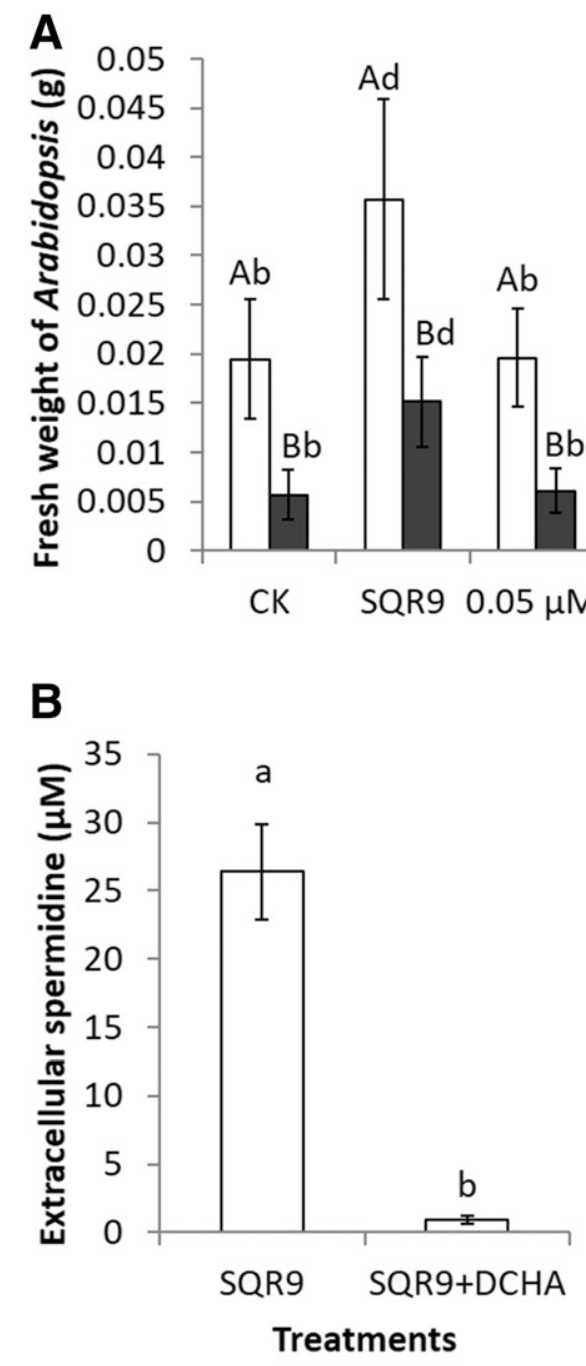

D
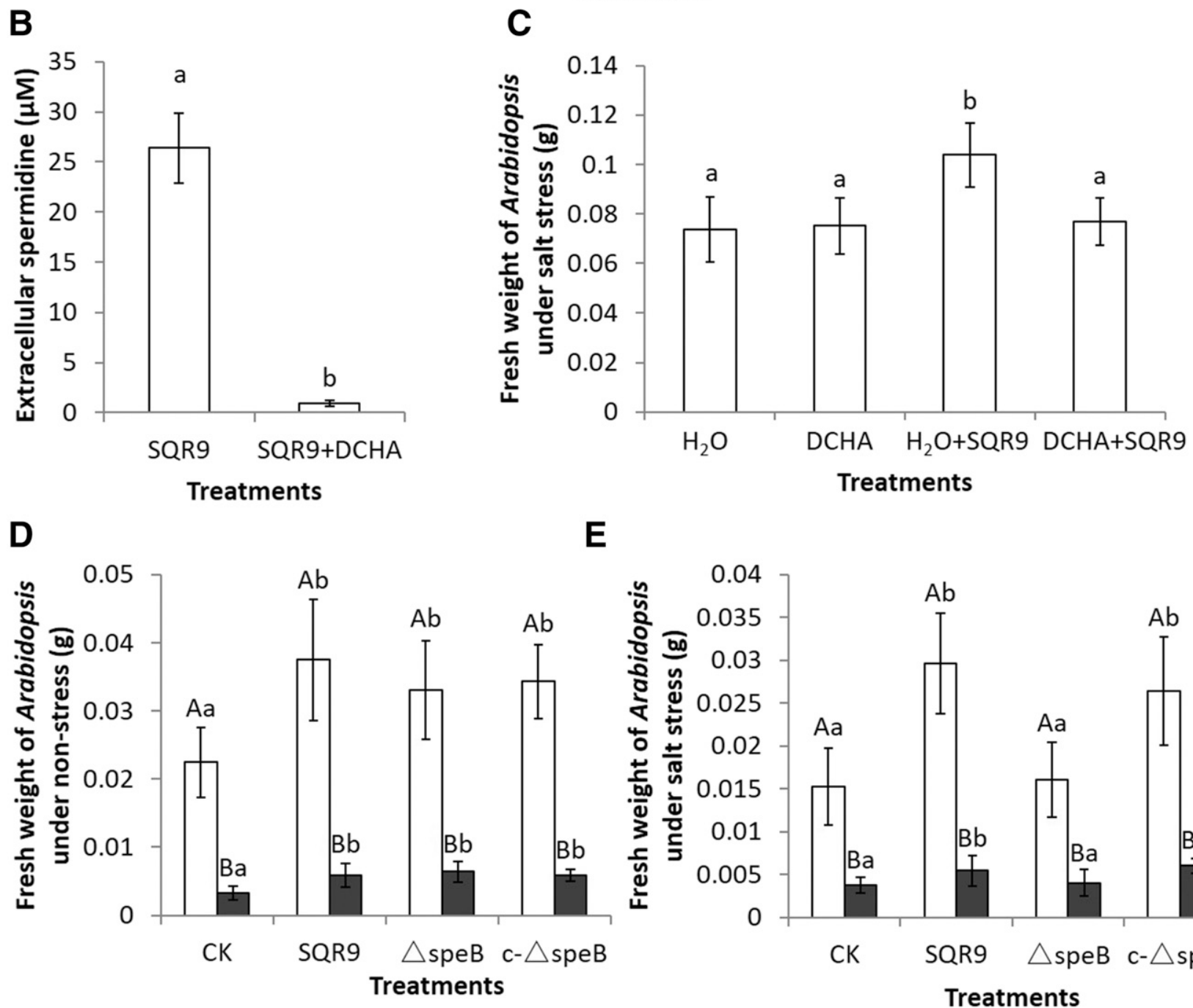

$\mathbf{E}$

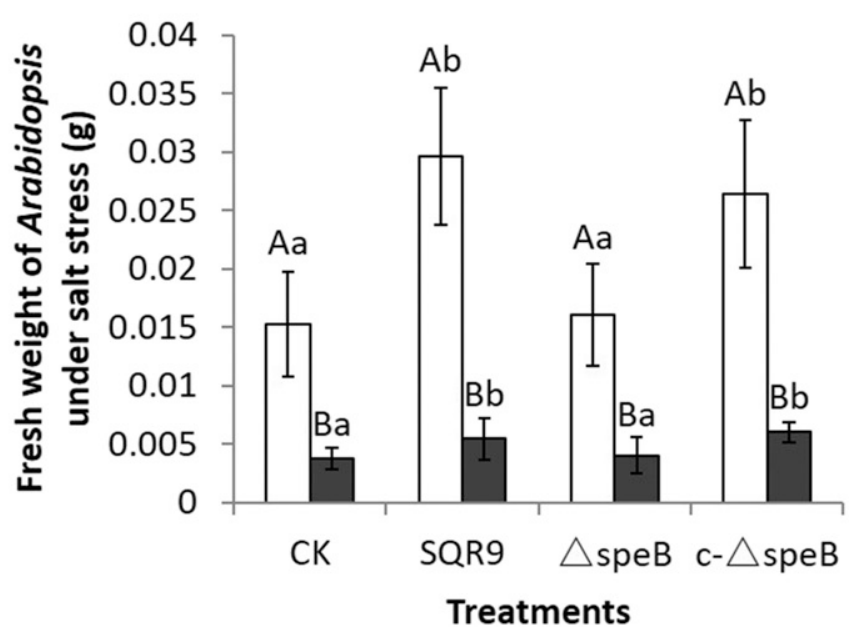

Fig. 6. A, Plant salt tolerance was affected by different concentrations of synthetic spermidine. Salt concentration is $100 \mathrm{mM}$ NaCl. $\mathrm{CK}=$ negative control. $\mathbf{B}$, Spermidine concentration of culture filtrates of wild-type SQR9 and SQR9 treated with dicyclohexylamine (DCHA). C, Effect of DCHA, an inhibitor of spermidine synthase, on plant salt tolerance. The fresh weight of Arabidopsis was measured after 14 days of treatment under salt stress. The fresh weight of Arabidopsis, treated with Bacillus amyloliquefaciens SQR9, $\Delta$ speB, and c- $\Delta$ speB strains and the heat-inactivated SQR9 control, was measured under D, nonstress and E, salt stress conditions. White and black bars represent shoot and root fresh weights, respectively. Multiple statistical analyses were performed in groups labeled with the same uppercase letter. Different lowercase letters above the columns indicate statistically significant differences (Duncan's least significant difference test, $P \leq 0.05, n=48$ ). 
shown to be the major signal that induced plant salt tolerance (Fig. 6E). The bacterially produced spermidine may induce systemic tolerance to salt stress by regulating the GSH content and $\mathrm{Na}^{+}$dynamics at the transcriptional level in plants. Overproduction of ROS induced by salt stress serves as a signal to regulate $\mathrm{Na}^{+}$homeostasis and causes damage to plants (Deinlein et al. 2014; Moschou et al. 2008). We suppose that the excess ROS caused by salt stress would not be reduced at the early stage of inoculation of SQR9. After salt stress for 14 days, ROS content would be reduced by the bacterial-produced spermidine due to the accumulation of GSH (Figs. 2 and 8).

Furthermore, spermidine regulates expression of NHX1 (the $\mathrm{Na}^{+}$transporters located at the vacuolar membrane) and SOS proteins to sequester $\mathrm{Na}^{+}$into vacuoles and expel $\mathrm{Na}^{+}$from the cell, thereby alleviating $\mathrm{Na}^{+}$toxicity (Figs. 2, 8, and 9). In the present study, the $\mathrm{Na}^{+}$contents of sos mutant plants were relatively higher compared with other reports $(24 \mathrm{~h})$ due to the longer treatment time with salt (14 days) (Zhang et al. 2008; Zhu et al. 1998). Zhu et al. (1998) reported that $\mathrm{Na}^{+}$content in sosl is significantly lower than that in $\operatorname{sos} 2$ and $\operatorname{sos} 3$ in the presence of salt stress but we did not detect the significant difference. The reason for the inconsistency might be the prolonged treatment time with salt, leading to damage of sosl (Shi et al. 2002). It is reported that SOS3 is necessary for the SOS pathway in roots but not in shoots (Deinlein et al. 2014; Quan et al. 2007). However, we found that both roots and shoots of $\operatorname{sos} 3$ showed deficiency in response to SQR9 under salt stress. It is believed that roots of $\operatorname{sos} 3$ would be damaged by salt stress in the presence of SQR9 and, consequently, the shoot would be affected by the impaired root. That would be the reason why the decrease of shoot biomass and increase of $\mathrm{Na}^{+}$content in shoots of $\operatorname{sos} 3$ could not be released by inoculation of SQR9.

Studies of the induction of systemic tolerance by $B$. subtilis GB03 showed that VOC emitted by GB03, such as 2,3-butanediol and acetoin (Farag et al. 2013), are the major compounds that help plants to tolerate abiotic stress (Zhang et al. 2008). Interestingly, the signal transduction conferring plant tolerance in the interaction between $B$. amyloliquefaciens SQR9 and plants is quite different from that in the B. subtilis GB03-plant interaction, because VOC emitted by SQR9 did not significantly improve plant salt tolerance (Supplementary Fig. S11). The VOC emitted from GB03 reduce $\mathrm{Na}^{+}$content in plants under salt stress by affecting the transcription of $\mathrm{HKT} 1, \mathrm{a} \mathrm{K}^{+}$transporter located in the plasma membrane of Arabidopsis that regulates $\mathrm{Na}^{+}$uptake and shoot-to-root $\mathrm{Na}^{+}$recirculation in Arabidopsis (Zhang et al. 2008). In addition, SOS3, the protein involved in the regulation of several ion transporters that respond to salt stress, is not required for the GB03 to induce plant tolerance of salt stress (Zhang et al. 2008). In contrast, we found that HKT1 is not required for the spermidine-induced Arabidopsis tolerance to salt stress. However, the NHX1 and SOS proteins are essential for exerting the function of SQR9-produced spermidine on plant salt tolerance. It is known that osmotic stress and ion toxicity are always exhibited in plants under salinity conditions (Deinlein et al. 2014; Zhu 2001). GB03 upregulated the expression of PEAMT to enhance choline and glycine betaine content, thereby helping Arabidopsis tolerate osmotic stress (Zhang et al. 2010). However, SQR9 does not upregulate PEAMT expression (data not shown).

In plants, the PA protect plants from salt stress, especially spermidine and spermine. The PA metabolic route interacts with several metabolisms such as biosynthesis of GSH, nitrogen metabolism, the SOS signal pathway, and others (Alcázar et al. 2010; Kusano et al. 2007, 2008). Previous studies have revealed that PA play a critical role in plant tolerance to abiotic stress (Takahashi and Kakehi 2010; Yamaguchi et al. 2006). Arabidopsis double-knockout mutant plants (acl5/spms), which could no longer produce spermine are highly sensitive to salt, whereas exogenous synthetic spermine mitigates plant salt hypersensitivity (Yamaguchi et al. 2006). TSP-16, the spermidine synthase overexpressed transgenic plants, showed upregulated expression of various genes related to stress and enhanced plant salt tolerance (Kasukabe et al. 2004).

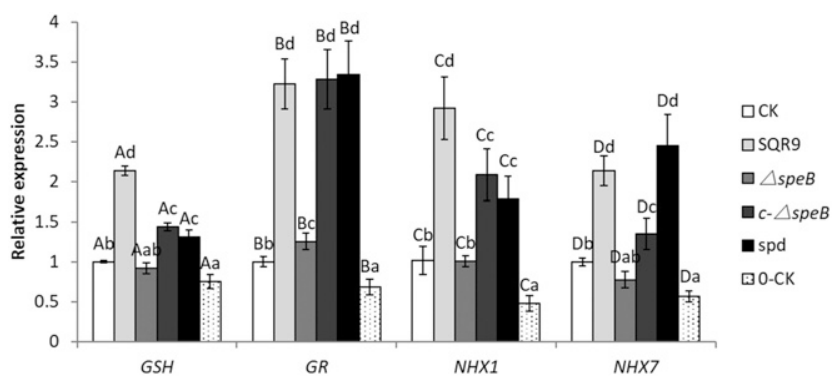

Fig. 7. Real-time quantitative polymerase chain reaction analysis of $G S, G R$, NHX1, and NHX7 transcript levels in Arabidopsis in response to Bacillus amyloliquefaciens $\mathrm{SQR} 9, \Delta$ speB, and $\mathrm{c}-\Delta s p e B$ strains, and $5 \mu \mathrm{M}$ spermidine (spd) under salt stress with $100 \mathrm{mM} \mathrm{NaCl}$. CK and $0-\mathrm{CK}=$ plants in response to inactive SQR9 under salt stress and in the absence of salt stress, respectively. Transcription levels were measured after plants were treated for 1 day under salt stress and compared with the control. Different letters indicate significant differences between treatments ( $t$ test, $n=3, P \leq 0.05$ ).

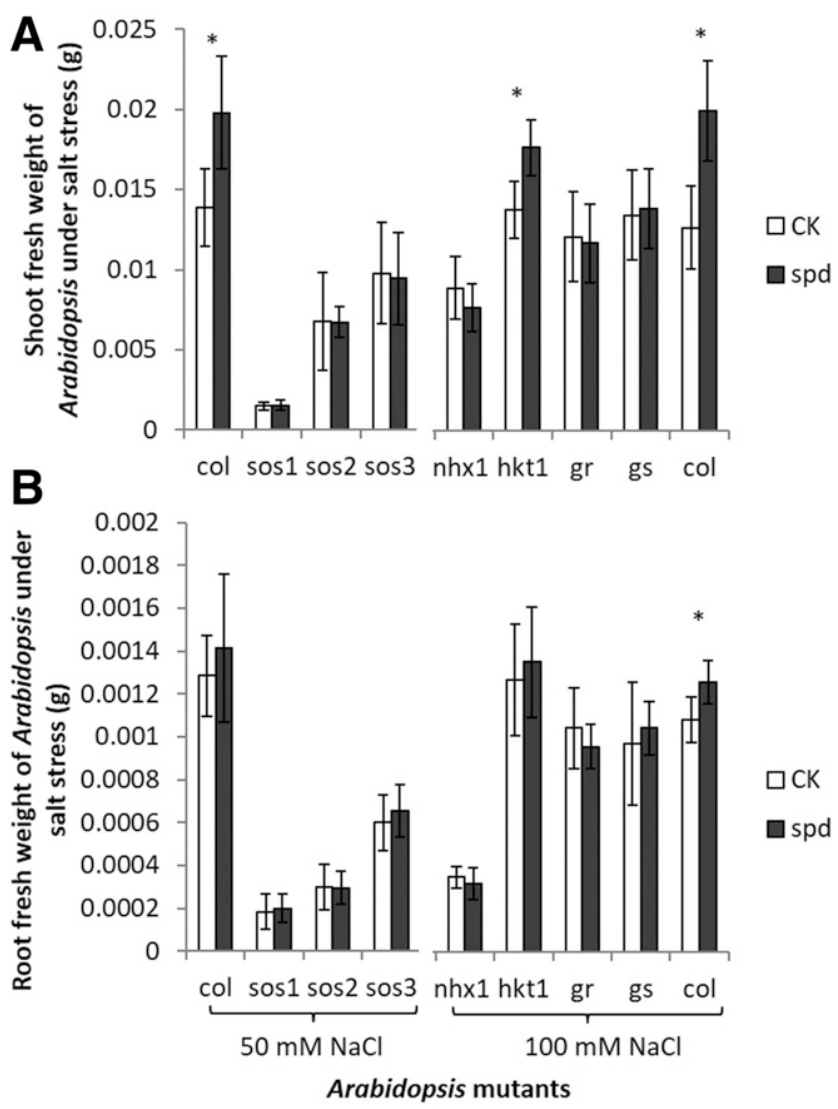

Fig. 8. Effects of $5 \mu \mathrm{M}$ spermidine (spd) on the response of Arabidopsis mutants to salt tolerance. A, Shoot fresh weight and B, root fresh weight of Arabidopsis were tested under salt stress for 14 days. White and black bars represent negative control and spermidine (spd) treatments, respectively. Columns: col-50 and col-100 = the wild type treated with 50 and $100 \mathrm{mM}$ $\mathrm{NaCl}$, respectively; $\operatorname{sos} 1$, sos 2 , and $\operatorname{sos} 3$ mutants were treated with $50 \mathrm{mM}$ $\mathrm{NaCl}$; and $n h x 1, h k t 1, g r$, and $g s$ mutants were treated with $100 \mathrm{mM} \mathrm{NaCl}$. Values are the mean \pm standard deviation from 48 replicates. An asterisk (*) indicates statistically significant differences between control plants and plants treated with spd ( $t$ test, $P \leq 0.05$ ). 
Spermidine can be synthesized by several Bacillus spp. (Burrell et al. 2010; Sekowska et al. 1998; Xie et al. 2014). B. subtilis OKB105 produces spermidine as a growth-promoting substance and promotes Nicotiana tabacum growth by regulating expansin expression and reducing ethylene content in plants (Xie et al. 2014). Xie et al. (2014) believe that the growth promotion of $N$. tabacum by OKB105 is achieved by the fact that bacterial spermidine enhanced the biofilm formation of OKB105, thereby enhancing the bacterial ecological fitness. The new finding of this study is that bacterially produced spermidine is more than a growth-promoting substance: it is also a critical molecule that induces maize and Arabidopsis salt tolerance (Fig. 6E).

In conclusion, this study demonstrates that spermidine produced by SQR9 is the key molecule that induces plant salt tolerance; accumulation of GSH and regulation of the SOS pathway are required during this process (Fig. 9). As a consequence of SOS regulation, $\mathrm{Na}^{+}$is sequestered into vacuoles and expelled from the cell to reduce $\mathrm{Na}^{+}$toxicity. In addition, GSH content is enhanced to reduce ROS damage during salt stress. However, the mechanism by which plants sense SQR9-produced spermidine is still not clear, and these questions merit further investigation in future studies.

\section{MATERIALS AND METHODS}

\section{Strains and plant culture conditions.}

B. amyloliquefaciens SQR9 (CGMCC accession number 5808; China General Microbiology Culture Collection Center) was cultured at $30^{\circ} \mathrm{C}$ with shaking at $170 \mathrm{rpm}$ for $10 \mathrm{~h}$ in $\mathrm{LB}$ medium (yeast extract at $5 \mathrm{~g} \mathrm{liter}^{-1}$, tryptone at $10 \mathrm{~g} \mathrm{liter}^{-1}$, and $\mathrm{NaCl}$ at $10 \mathrm{~g} \mathrm{liter}^{-1} ; \mathrm{pH} 7.0$ to 7.2). One culture was adequately hyperthermia inactivated twice and used as a "dead cell" negative control. The cultures were centrifuged at $6,000 \mathrm{rpm}$ for $10 \mathrm{~min}$. The cells were suspended and diluted with doubledistilled water to a final concentration of $5 \times 10^{6} \mathrm{CFU} \mathrm{m}{ }^{-1}$.

Maize (Zea mays 'Jingtian') seed were sterilized and germinated in a growth chamber. Three-day-old seedlings were transplanted into sterilized one-quarter-strength (1/4) Hoagland medium (Hoagland and Arnon 1938) and grown at $25^{\circ} \mathrm{C}$ in a growth chamber with a photoperiod of $16 \mathrm{~h}$ of light and $8 \mathrm{~h}$ of darkness. The medium was shaken at $50 \mathrm{rpm}$ for $2 \mathrm{~h}$ every day and replaced every 2 days (Chen et al. 2016).

Arabidopsis thaliana (col-0) seed were surface sterilized and then placed on one-half-strength (1/2) MS media ( $\mathrm{pH}$ 5.7) containing $1.5 \%$ sucrose and $0.8 \%$ agar (Murashige and Skoog 1962). T-DNA insertion mutants were obtained from The Arabidopsis Information Resource (TAIR; http://www.arabidopsis.org) and identified in the SALK and GABI-KAT databases. The mutants used in this study were as follows: $g s, g r, n h x 1, \operatorname{sos} 1, \operatorname{sos} 2, \operatorname{sos} 3$,

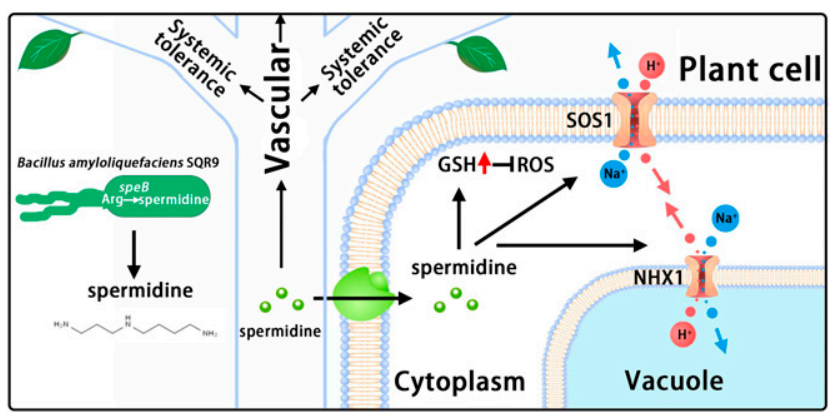

Fig. 9. Bacillus amyloliquefaciens SQR9 induces systemic tolerance to salt stress in plants. Spermidine produced by SQR9 enhances reduced glutathione (GSH) content, thus reducing reactive oxygen species (ROS) content, and regulates the salt overly sensitive (SOS) pathway to sequester $\mathrm{Na}^{+}$into vacuoles and expel $\mathrm{Na}^{+}$from the cytoplasm to confer plant salt tolerance. and hktl (Table 1). Arabidopsis mutant seed from the $\mathrm{T}_{2}$ progeny were sterilized and germinated as described above. Gene-specific primer pairs and T-DNA-specific primers were used for polymerase chain reaction (PCR) screening to identify T-DNA insertions.

\section{Inoculation of strains.}

Arabidopsis seed were vernalized at $4^{\circ} \mathrm{C}$ for 2 days in the dark and then incubated at $22^{\circ} \mathrm{C}$ in a growth chamber with a photoperiod of $16 \mathrm{~h}$ of light and $8 \mathrm{~h}$ of darkness. Seven-day-old seedlings were transplanted to $1 / 2 \mathrm{MS}$ solid medium with or without $100 \mathrm{mM} \mathrm{NaCl}$. To measure the impact of SQR9 on plant tolerance, each plate was inoculated with $2 \mu \mathrm{l}$ of active SQR9 or dead cell suspension. The $n h x 1, \operatorname{sos} 1, \operatorname{sos} 2$, and $\operatorname{sos} 3$ mutants were transplanted onto medium with or without $50 \mathrm{mM}$ $\mathrm{NaCl}$. Ten plates were included in each treatment and six plants were included in each plate to control for variability. In each plate, the left three plants were mutants and the right three plants were the wild type.

Ten-day-old Arabidopsis seedlings were detached from 1/2 MS agar plates and transplanted onto 1/4 MS liquid medium. Thirteen-day-old Arabidopsis and maize seedlings were treated with or without $100 \mathrm{mM} \mathrm{NaCl}$ and inoculated with bacterial suspensions in different MWCO dialysis bags (100, 500, 1,000, 2,000 , or $5,000 \mathrm{MWCO}$ ). In this experiment, bacteria cells were suspended with 1/4 MS medium and 1/4 Hoagland medium, respectively. The media were shaken at $50 \mathrm{rpm}$ for $2 \mathrm{~h}$ every day and replaced every 2 days. Bacterial suspension also was changed every 2 days. The experiments were repeated three times. For each treatment, 48 Arabidopsis and 21 maize seedlings were included as replicates.

\section{Determination of plant biomass.}

The plant fresh weight was measured by an analytical scale. Plant height and root length were measured with a ruler. Lateral roots were observed and counted with a stereomicroscope.

\section{Determination of leaf chlorophyll, reduced GSH, and $\mathrm{Na}^{+}$content.}

Chlorophyll content (SPAD value) was measured using a chlorophyll meter (SPAD-502) (Ling et al. 2011). The leaves from different plants used for detection were in the same location on plants. For each treatment, 10 independent samples were measured.

The enzymatic recycling method used to measure GSH was previously described (Airaki et al. 2011; Griffith 1980).

To determine $\mathrm{Na}^{+}$content, whole plants were washed with deionized water and dried at $70^{\circ} \mathrm{C}$ for 2 days. All plant samples were homogenized and filtered using a filter with a 1-mm pore

Table 1. Arabidopsis mutants used in this study

\begin{tabular}{|c|c|c|c|}
\hline Mutants & Stock name & Locus & Function \\
\hline gs & SALK_081530C & At4g23100 & $\begin{array}{l}\text { Encodes the enzyme } \\
\text { glutamate-cysteine ligase }\end{array}$ \\
\hline$g r$ & SALK_104319C & At3g24170 & $\begin{array}{l}\text { Encodes glutathione-disulfide } \\
\text { reductase }\end{array}$ \\
\hline$n h x 1$ & SALK_034001C & At5g27150 & $\begin{array}{l}\text { Encodes } \mathrm{Na}^{+} / \mathrm{H}^{+} \text {antiporters } \\
\text { in the tonoplast }\end{array}$ \\
\hline $\operatorname{sos} 1$ & SALK_092851C & At2g01980 & $\begin{array}{l}\text { Encodes } \mathrm{Na}^{+} / \mathrm{H}^{+} \text {antiporters } \\
\text { in the plasma membrane, } \\
\text { SOS } 1\end{array}$ \\
\hline $\operatorname{sos} 2$ & SALK_000367C & At5g01820 & Encodes protein kinase, SOS2 \\
\hline $\operatorname{sos} 3$ & SALK_110426C & At4g17615 & $\begin{array}{l}\text { Encodes calcineurin B-like } \\
\text { protein, SOS } 3\end{array}$ \\
\hline hkt1 & CS476306 & At4g10310 & $\begin{array}{l}\text { Encodes high-affinity } \mathrm{K}^{+} \\
\text {transporter } 1\end{array}$ \\
\hline cat2-1 & SALK_076998 & AT4G35090 & Encodes catalase 2 \\
\hline
\end{tabular}


size. The $\mathrm{Na}^{+}$in the plants was extracted as described by Zhang and quantified using an inductively coupled plasma spectrograph (Zhang et al. 2008).

\section{Fractionation of bacterial secretions.}

SQR9 was cultured in Landy medium (Landy et al. 1948) at $30^{\circ} \mathrm{C}$ for $36 \mathrm{~h}$. Filtered SQR9 secretions and Landy medium (negative control) were freeze dried and then dissolved in $50 \mathrm{ml}$ of sterile distilled water. Then, the 100- to 500-Da SQR9 secretions and Landy medium were collected using 100- and 500-Da MWCO dialysis bags. The 100- to 500-Da SQR9 secretions $(50 \mathrm{ml})$ and Landy medium were partitioned with $50 \mathrm{ml}$ of ethyl acetate and the ethyl acetate phase was separated. Then, the aqueous phase was fractionated with $50 \mathrm{ml}$ of ethyl ether and the ethyl ether phase was separated. The aqueous phase was fractionated with $50 \mathrm{ml}$ of n-pentane and the n-pentane phase was separated. Finally, the aqueous phase was fractionated with $50 \mathrm{ml}$ of chloroform and the chloroform and aqueous phases were separated. Each organic phase was dried under nitrogen gas. This process resulted in five types of fractions: ethyl acetate, ethyl ether, n-pentane, chloroform, and aqueous fractions. The SQR9 secretion without fractionation (total secretion sample) was also kept.

\section{Construction of a $B$. amyloliquefaciens SQR9 mutant strain.}

To confirm the contribution of SQR9-derived spermidine to plant salt tolerance, the speB gene was completely deleted by double cross-over (Yan et al. 2008; Zhou et al. 2017). The sequences of erythromycin were obtained from pAX01 plasmid. Two partial sequence fragments of the $s p e B$ gene were amplified from SQR9 DNA. Then, the recombinant fragments were fused and transformed into the SQR9 strain to generate the $\Delta s p e B$ mutation. To complement the $\Delta s p e B$ mutant, the entire $s p e B$ gene was amplified from $B$. amyloliquefaciens SQR9 chromosomal DNA and ligated into the pNW33 plasmid to obtain pNW33-speB, which was transformed into the $\Delta$ speB mutant to get the complementary strain of mutant $\Delta$ speB (c- $\Delta$ spe $B)$. The primers used to construct these mutants are shown in Supplementary Table S1.

\section{Measurement of spermidine.}

The bacterial strains were cultured in Landy medium at $30^{\circ} \mathrm{C}$ for $36 \mathrm{~h}$. The fermented cultures were centrifuged at $6,000 \mathrm{rpm}$ for $10 \mathrm{~min}$ at $4^{\circ} \mathrm{C}$. The bacterial cells were treated with lysozyme and suspended in $0.1 \mathrm{M} \mathrm{HCl}$, then sonicated. Pure perchloric acid was added to culture filtrates or lysates to obtain a $5 \%(\mathrm{wt} / \mathrm{vol})$ solution for $1 \mathrm{~h}$ at $4^{\circ} \mathrm{C}$. The samples were centrifuged at 10,000 rpm for $30 \mathrm{~min}$. Benzoyl chloride $(70 \mu \mathrm{l})$ was added to $5 \mathrm{ml}$ of supernatant. Then, each sample was neutralized with $10 \mathrm{ml}$ of $2 \mathrm{M} \mathrm{NaOH}$, vortexed for $20 \mathrm{~s}$, and incubated at $37^{\circ} \mathrm{C}$ for $30 \mathrm{~min}$. A saturated $\mathrm{NaCl}$ solution and ethyl ether $(15 \mathrm{ml})$ were added to each sample. The organic phase was dried using nitrogen and resuspended in $200 \mu \mathrm{l}$ of methanol. The sample was detected by an HPLC system equipped with a UV detector set at $254 \mathrm{~nm}$ and a C18 column (Waters). The mobile phase was $60 \%$ methanol, the flow rate was $0.7 \mathrm{ml}$ $\min ^{-1}$ and the column temperature was $30^{\circ} \mathrm{C}$.

To detect the role of spermidine in plant salt tolerance, different concentrations of exogenous spermidine were added to MS medium with $100 \mathrm{mM} \mathrm{NaCl}$, and $10 \mathrm{mM} \mathrm{DCHA}$ (an inhibitor of PA biosynthesis) was added to the bacterial growth medium. Each treatment had six plates containing six plants each.

\section{Transcription analysis.}

RNA was extracted from plants after 1 day of treatment. Plant tissue was flash frozen in liquid nitrogen and then extracted using the Qiagen RNeasy plant mini kit (Qiagen). The transcript levels were quantified by reverse transcription polymerase chain reaction using the PrimeScript RT reagent Kit (TaKaRa Dalian Biotechnology). Quantitative real-time (qRT)PCR assays were performed with SYBR Premix EX Taq (TaKaRa Dalian Biotechnology) using an ABI 7500 Cycler (Applied Biosystems). Transcription levels of $G S$ (encoding GS), GR (encoding GR), NHXI (encoding $\mathrm{Na}^{+} / \mathrm{H}^{+}$antiporters in the tonoplast), and $N H X 7$ (encoding $\mathrm{Na}^{+} / \mathrm{H}^{+}$antiporters in the plasma membrane) were measured using $A C T$ (encoding actin) as an internal reference. For each treatment, three biological replicates are included. The specificity of the amplification was verified by melting-curve analysis and agarose gel electrophoresis. The relative transcription levels were calculated using the $2^{-\Delta \Delta C T}$ method (Livak and Schmittgen 2001).

\section{Statistical analysis.}

The data among different treatments were subjected to analysis of variance. Duncan's multiple range tests $(P<0.05)$ was employed to determine differences among means. Statistical significance was analyzed by $t$ test and SPSS (version 18.0; SPSS, Inc.).

\section{Accession numbers.}

The sequences of genes used in this article can be found in National Center for Biotechnology Information or TAIR data library under the following accession numbers: Act (At3g18780), NHX1 (At5g27150), NHX7 (At2g01980), GR (At3g24170), and GS (At5g35630).

\section{ACKNOWLEDGMENTS}

This work was financially supported by National Natural Science Foundation of China (31330069, 31600088, and 31572214), the National Key Basic Research Program of China (973 program, 2015CB150505), the National Key Research and Development Program (2016YFE0101100 and 2016YFD0200300), the Fundamental Research Funds for the Central Non-profit Research Institution of the Chinese Academy of Forestry (CAFYBB2017MA020), and China Postdoctoral Science Foundation (2016M591297). R. Zhang and Q. Shen were also supported by the Key Projects of International Cooperation in Science and Technology Innovation (2016YFE0101100), the 111 Project (B12009), and the Priority Academic Program Development of Jiangsu Higher Education Institutions. We thank J. Shao and H. Feng (Nanjing Agricultural University) for excellent technical assistance and Y. Ren for excellent care of plants.

\section{LITERATURE CITED}

Airaki, M., Sánchez-Moreno, L., Leterrier, M., Barroso, J. B., Palma, J. M., and Corpas, F. J. 2011. Detection and quantification of S-nitrosoglutathione (GSNO) in pepper (Capsicum annuиm L.) plant organs by LC-ES/MS. Plant Cell Physiol. 52:2006-2015.

Alcázar, R., Altabella, T., Marco, F., Bortolotti, C., Reymond, M., Koncz, C., Carrasco, P., and Tiburcio, A. F. 2010. Polyamines: Molecules with regulatory functions in plant abiotic stress tolerance. Planta 231:1237-1249.

Biondi, S., Torrigiani, P., Sansovini, A., Bagni, N., and Inhibition, N. 1988. Inhibition of polyamine biosynthesis by dicyclohexylamine in cultured cotyledons of Pinus radiata. Physiol. Plant. 72:471-476.

Burrell, M., Hanfrey, C. C., Murray, E. J., Stanley-Wall, N. R., and Michael, A. J. 2010. Evolution and multiplicity of arginine decarboxylases in polyamine biosynthesis and essential role in Bacillus subtilis biofilm formation. J. Biol. Chem. 285:39224-39238.

Chen, L., Liu, Y., Wu, G., Veronican Njeri, K., Shen, Q., Zhang, N., and Zhang, R. 2016. Induced maize salt tolerance by rhizosphere inoculation of Bacillus amyloliquefaciens SQR9. Physiol. Plant. 158:34-44.

Chinnusamy, V., Zhu, J., and Zhu, J.-K. 2006. Salt stress signaling and mechanisms of plant salt tolerance. Pages 141-177 in: Genetic Engineering: Principles and Methods, Vol. 27. J. K. Setlow, ed. Springer US, New York.

Deinlein, U., Stephan, A. B., Horie, T., Luo, W., Xu, G., and Schroeder, J. I. 2014. Plant salt-tolerance mechanisms. Trends Plant Sci. 19:371-379.

Dimkpa, C., Weinand, T., and Asch, F. 2009. Plant-rhizobacteria interactions alleviate abiotic stress conditions. Plant Cell Environ. 32:1682-1694. 
Dinneny, J. R., Long, T. A., Wang, J. Y., Jung, J. W., Mace, D., Pointer, S., Barron, C., Brady, S. M., Schiefelbein, J., and Benfey, P. N. 2008. Cell identity mediates the response of Arabidopsis roots to abiotic stress. Science 320:942-945.

Dodd, I. C., and Pérez-Alfocea, F. 2012. Microbial amelioration of crop salinity stress. J. Exp. Bot. 63:3415-3428.

Fan, B., Carvalhais, L. C., Becker, A., Fedoseyenko, D., von Wirén, N., and Borriss, R. 2012. Transcriptomic profiling of Bacillus amyloliquefaciens FZB42 in response to maize root exudates. BMC Microbiol. 12:116-128.

Farag, M. A., Zhang, H., and Ryu, C. M. 2013. Dynamic chemical communication between plants and bacteria through airborne signals: Induced resistance by bacterial volatiles. J. Chem. Ecol. 39:1007-1018.

Geng, Y., Wu, R., Wee, C. W., Xie, F., Wei, X., Chan, P. M., Tham, C., Duan, L., and Dinneny, J. R. 2013. A spatio-temporal understanding of growth regulation during the salt stress response in Arabidopsis. Plant Cell 25:2132-2154

Glick, B. R., Todorovic, B., Czarny, J., Cheng, Z., Duan, J., and McConkey, B. 2007. Promotion of plant growth by bacterial ACC deaminase. CRC. Crit. Rev. Plant Sci. 26:227-242.

Griffith, O. W. 1980. Determination of glutathione and glutathione disulfide using glutathione reductase and 2-vinylpyridine. Anal. Biochem. 106: 207-212.

Hoagland, D., and Arnon, D. I. 1938. The water culture method for growing plants without soil. Calif. Agric. Exp. Stn. Bull. 347:1-39.

Kasukabe, Y., He, L., Nada, K., Misawa, S., Ihara, I., and Tachibana, S. 2004. Overexpression of spermidine synthase enhances tolerance to multiple environmental stresses and up-regulates the expression of various stress-regulated genes in transgenic Arabidopsis thaliana. Plant Cell Physiol. 45:712-722.

Kusano, T., Berberich, T., Tateda, C., and Takahashi, Y. 2008. Polyamines: Essential factors for growth and survival. Planta 228:367-381.

Kusano, T., Yamaguchi, K., Berberich, T., and Takahashi, Y. 2007. The polyamine spermine rescues Arabidopsis from salinity and drought stresses. Plant Signal. Behav. 2:251-252.

Landy, M., Warren, G. H., Rosenman, S. B., and Colio, L. G. 1948. Bacillomycin: An antibiotic from Bacillus subtilis active against pathogenic fungi. Proc. Soc. Exp. Biol. Med. 67:539-541.

Ling, Q., Huang, W., and Jarvis, P. 2011. Use of a SPAD-502 meter to measure leaf chlorophyll concentration in Arabidopsis thaliana. Photosynth. Res. 107:209-214.

Liu, X.-M., and Zhang, H. 2015. The effects of bacterial volatile emissions on plant abiotic stress tolerance. Front. Plant Sci. 6:774.

Livak, K. J., and Schmittgen, T. D. 2001. Analysis of relative gene expression data using real-time quantitative PCR and the $2^{-\Delta \triangle C T}$ method. Methods 25:402-408.

Ma, Y., He, J., Ma, C., Luo, J., Li, H., Liu, T., Polle, A., Peng, C., and Luo, Z.-B. 2014. Ectomycorrhizas with Paxillus involutus enhance cadmium uptake and tolerance in Populus $\times$ canescens. Plant Cell Environ. 37: 627-642.

Mayak, S., Tirosh, T., and Glick, B. R. 2004. Plant growth-promoting bacteria that confer resistance to water stress in tomatoes and peppers. Plant Sci. 166:525-530.

Meyer, A. J., Brach, T., Marty, L., Kreye, S., Rouhier, N., Jacquot, J. P., and Hell, R. 2007. Redox-sensitive GFP in Arabidopsis thaliana is a quantitative biosensor for the redox potential of the cellular glutathione redox buffer. Plant J. 52:973-986.

Mittler, R., and Blumwald, E. 2015. The roles of ROS and ABA in systemic acquired acclimation. Plant Cell 27:64-70.

Moschou, P. N., Paschalidis, K. A., Delis, I. D., Andriopoulou, A. H., Lagiotis, G. D., Yakoumakis, D. I., and Roubelakis-Angelakis, K. A. 2008. Spermidine exodus and oxidation in the apoplast induced by abiotic stress is responsible for $\mathrm{H}_{2} \mathrm{O}_{2}$ signatures that direct tolerance responses in tobacco. Plant Cell 20:1708-1724.

Murashige, T., and Skoog, F. 1962. A revised medium for rapid growth and bio assays with tobacco tissue cultures. Physiol. Plant. 15:473-497.

Quan, R., Lin, H., Mendoza, I., Zhang, Y., Cao, W., Yang, Y., Shang, M., Chen, S., Pardo, J. M., and Guo, Y. 2007. SCABP8/CBL10, a putative calcium sensor, interacts with the protein kinase SOS2 to protect Arabidopsis shoots from salt stress. Plant Cell 19:1415-1431.

Rudrappa, T., Czymmek, K. J., Paré, P. W., and Bais, H. P. 2008. Rootsecreted malic acid recruits beneficial soil bacteria. Plant Physiol. 148: $1547-1556$

Sekowska, A., Bertin, P., and Danchin, A. 1998. Characterization of polyamine synthesis pathway in Bacillus subtilis 168. Mol. Microbiol. 29:851-858.

Shabala, S., and Cuin, T. A. 2008. Potassium transport and plant salt tolerance. Physiol. Plant. 133:651-669.

Shi, H., Quintero, F. J., Pardo, J. M., and Zhu, J. K. 2002. The putative plasma membrane $\mathrm{Na}^{+} / \mathrm{H}^{+}$antiporter $\mathrm{SOS}_{1}$ controls long-distance $\mathrm{Na}^{+}$ transport in plants. Plant Cell 14:465-477.

Suárez, R., Wong, A., Ramírez, M., Barraza, A., del Carmen Orozco, M., Cevallos, M. A., Lara, M., Hernández, G., and Iturriaga, G. 2008 Improvement of drought tolerance and grain yield in common bean by overexpressing trehalose-6-phosphate synthase in rhizobia. Mol. PlantMicrobe Interact. 21:958-966.

Székely, G., Ábrahám, E., Cséplő, A., Rigó, G., Zsigmond, L., Csiszár, J., Ayaydin, F., Strizhov, N., Jásik, J., Schmelzer, E., Koncz, C., and Szabados, L. 2008. Duplicated P5CS genes of Arabidopsis play distinct roles in stress regulation and developmental control of proline biosynthesis. Plant J. 53:11-28.

Takahashi, T., and Kakehi, J. 2010. Polyamines: Ubiquitous polycations with unique roles in growth and stress responses. Ann. Bot. (Lond.) 105:1-6.

Upadhyay, S. K., Singh, J. S., Saxena, A. K., and Singh, D. P. 2012. Impact of PGPR inoculation on growth and antioxidant status of wheat under saline conditions. Plant Biol. 14:605-611.

Xie, S. S., Wu, H. J., Zang, H. Y., Wu, L. M., Zhu, Q. Q., and Gao, X. W. 2014. Plant growth promotion by spermidine-producing Bacillus subtilis OKB105. Mol. Plant-Microbe Interact. 27:655-663.

Yamaguchi, K., Takahashi, Y., Berberich, T., Imai, A., Miyazaki, A., Takahashi, T., Michael, A., and Kusano, T. 2006. The polyamine spermine protects against high salt stress in Arabidopsis thaliana. FEBS Lett. 580: 6783-6788.

Yan, X., Yu, H. J., Hong, Q., and Li, S. P. 2008. Cre/lox system and PCRbased genome engineering in Bacillus subtilis. Appl. Environ. Microbiol. 74:5556-5562.

Yang, J., Kloepper, J. W., and Ryu, C. M. 2009. Rhizosphere bacteria help plants tolerate abiotic stress. Trends Plant Sci. 14:1-4.

Zhang, H., Kim, M. S., Sun, Y., Dowd, S. E., Shi, H., and Paré, P. W. 2008. Soil bacteria confer plant salt tolerance by tissue-specific regulation of the sodium transporter HKT1. Mol. Plant-Microbe Interact. 21:737-744.

Zhang, H., Murzello, C., Sun, Y., Kim, M. S., Xie, X., Jeter, R. M., Zak, J. C., Dowd, S. E., and Paré, P. W. 2010. Choline and osmotic-stress tolerance induced in Arabidopsis by the soil microbe Bacillus subtilis (GB03). Mol. Plant-Microbe Interact. 23:1097-1104.

Zhou, C., Shi, L., Ye, B., Feng, H., Zhang, J., Zhang, R., and Yan, X. 2017. pheS*, an effective host-genotype-independent counter-selectable marker for marker-free chromosome deletion in Bacillus amyloliquefaciens. Appl. Microbiol. Biotechnol. 101:217-227.

Zhu, J.-K. 2001. Plant salt tolerance. Trends Plant Sci. 6:66-71.

Zhu, J.-K., Liu, J., and Xiong, L. 1998. Genetic analysis of salt tolerance in Arabidopsis: Evidence for a critical role of potassium nutrition. Plant Cell 10:1181-1191. 